\title{
A FUNDAMENTAL GROUP TOPOLOGY
}

\author{
C. H. HOUGHTON
}

(Received Dec 24, 1982)

Communicated by J. H. Rubinstein

\begin{abstract}
The topology of the Čech fundamental group of the one-point compactification of an appropriate space $Y$ induces a topology on the fundamental group of $Y$. We describe this topology in terms of a topological group introduced by Higman.
\end{abstract}

1980 Mathematics subject classification (Amer. Math. Soc.): 22 A 05, 55 Q 07.

\section{Introduction}

A free group on a topological space $X$ can be given various topologies, for example the discrete topology or the topology of the free topological group on $X$. Here we define an intermediate topology on the free group $F=F(X)$ when the space $X$ is 0 -dimensional and Hausdorff. In the case where $X$ is the one-point compactification $N^{*}$ of a countably infinite discrete space $N$, the group obtained coincides with a topological group $F^{(\omega)}$ studied by Higman (1952).

We are interested in the natural occurrence of the topological group $F$ in the context of Čech homotopy theory. The embedding of an infinite connected locally finite $C W$-complex $Y$ in its one-point compactification $Y^{*}$ gives rise to a homomorphism from the fundamental group $\pi(Y)$ to the Cech fundamental group $\check{\pi}\left(Y^{*}\right)$. Our aim is to identify the fundamental group topology thus induced on $\pi(Y)$. Using combinatorial methods, we show that $\pi(Y)$ is the continuous image of an epimorphism from $F^{(\omega)}$. In the 1-dimensional case, where $Y$ is a graph, it follows that $\check{\pi}\left(Y^{*}\right)$ is the completion of a topological free group on the one-point union of $N^{*}$ and the end space $E$ of $Y$.

(C) 1984 Australian Mathematical Society $0263-6115 / 84 \$ A 2.00+0.00$ 


\section{A free group topology}

Let $X$ be a 0-dimensional Hausdorff space with $x \in X$ chosen as basepoint and let $F=F(X, x)$ be the free group on $X \backslash\{x\}$. Every based map from $X$ to a discrete group extends to a homomorphism of $F$ and we give $F$ the coarsest topology which makes all such extensions continuous. We note some properties of this topological group.

THEOREM 1. The group $F=F(X, x)$ is 0-dimensional and Hausdorff and the injection from $X$ to $F$ is continuous. Any based map from $X$ to a discrete group $G$ lifts to a continuous homomorphism from $F$ to $G$.

Proof. A subbase for the topology on $F$ is given by the inverse images of elements of discrete groups, with respect to the specified maps. Such images are both open and closed and so $F$ is 0 -dimensional. To show that $F$ is Hausdorff, suppose that $v, w \in F$ with $v \neq w$ and that the elements of $X$ appearing in the words $v$ and $w$ are $x_{1}, \ldots, x_{n}$. There is a partition of $X$ into open sets $X_{0}, X_{1}, \ldots, X_{n}$ such that $x_{i} \in X_{i}$ and $x \in X_{0}$. Taking $G$ as the free group on $y_{1}, \ldots, y_{n}$ and putting $y_{0}=1$, the map $X_{i} \rightarrow y_{i}$ extends to a homomorphism $F \rightarrow G$ under which $v$ and $w$ have distinct images and thus are separated by disjoint open sets. The continuity of the injection follows from the definition of the topology on $F$, as does the final property.

The constancy sets of a map from $X$ to a discrete group form a partition of $X$ into open sets and, conversely, any such partition can be realised by a map to an appropriate free group. The topology on $F$ is thus given by homomorphisms mapping the parts of such partitions to distinct elements of discrete groups, with the part containing $x$ mapped to 1 . For example, let $X$ be the one-point compactification of the countably infinite discrete set $N=\left\{x_{1}, x_{2}, \ldots\right\}$, the additional point being $x$. Then the continuous homomorphisms of $F$ are precisely those mapping almost all $x_{i}$ to 1 . This topological group was introduced by Higman (1952) and is denoted by $F^{(\omega)}$.

If $X$ is compact, it is an inverse limit of the finite discrete spaces arising as quotient spaces of finite partitions of $X$ into open sets. We write $X=\lim _{\leftarrow} P_{\alpha}$, where $\alpha$ runs through all such partitions and $P_{\alpha}$ denotes the corresponding quotient space. There is an associated inverse system of discrete free groups $F\left(P_{\alpha}, p_{\alpha}\right)$ of finite rank, where $p_{\alpha}$ is the projection of $x$ to $P_{\alpha}$ and the maps are induced by inclusion. We define $C(X, x)$ to be the topological group $\underset{\leftarrow}{\leftarrow} F\left(P_{\alpha}, p_{\alpha}\right)$. 
TheOREM 2. For compact $X$, the group $C(X, x)$ is the completion of $F(X, x)$.

Proof. For given $\alpha$, let $M_{\alpha}$ be the kernel of the epimorphism $F(X, x) \rightarrow$ $F\left(P_{\alpha}, p_{\alpha}\right)$ induced by the projection map $X \rightarrow P_{\alpha}$. The topology on $F$ is the normal subgroup topology given by the $M_{\alpha}$ and $C(X, x)=\underset{\leftarrow}{\lim } F\left(P_{\alpha}, p_{\alpha}\right)$ $\cong \lim _{\leftarrow} F / M_{\alpha}$, which is the completion of the Hausdorff group $F$.

We remark that the paper of Higman (1952) is mainly concerned with properties of the completion of $F^{(\omega)}$.

\section{Identifying the fundamental group topology}

Suppose that $Y^{*}$ is the one-point compactification of an infinite connected locally finite $C W$-complex $Y$. We establish connections between groups of the type studied above and the Čech fundamental group $\check{\pi}\left(Y^{*}\right)$.

We summarise the results of Houghton (1981) which lead to a determination of $\check{\pi}\left(Y^{*}\right)$. Choosing a chain $Y=Y_{0} \supseteq Y_{1} \supseteq \cdots$ of cofinite subcomplexes of $Y$ with $\cap Y_{i}=\varnothing$, we have $Y^{*}=\lim Y / Y_{i}$ and then $\check{\pi}\left(Y^{*}\right)=\lim \pi\left(Y / Y_{i}, y_{i}\right)$, where $y_{i}$ is the projection of a chosen basepoint $y$ of $Y$. We put $\pi(Y, y)=H$ and choose an element $e$ of the end space $E=\lim \pi_{0}\left(Y_{i}\right)$ of $Y$. Standard methods show that $\pi\left(Y / Y_{i}, y_{i}\right)$ has the form $\left(H / H_{i}\right) * F_{i}$, where $H_{i}$ is the kernel of the homomorphism induced by the map $Y \rightarrow Y / Y_{i}$ and $F_{i}$ is the free group on the based set $\pi_{0}\left(Y_{i}\right)$, with the projection of $e$ as basepoint. The homomorphism from $\left(H / H_{i+1}\right) * F_{i+1}$ to $\left(H / H_{i}\right) * F_{i}$ is given by the natural map $H / H_{i+1} \rightarrow H / H_{i}$ together with the map $F_{i+1} \rightarrow F_{i}$ corresponding to $\pi_{0}\left(Y_{i+1}\right) \rightarrow \pi_{0}\left(Y_{i}\right)$. If $M$ denotes $\cap H_{i}$ then, in the case $M=H$, we have $\check{\pi}\left(Y^{*}, y\right)=\lim _{\leftarrow} F_{i}$, which is the completion $C(E, e)$ of $F(E, e)$. In the general case, $\check{\pi}\left(Y^{*}\right)$ is the completion of the Hausdorff group $(H / M) * F(E, e)$, where $H / M$ has the normal subgroup topology given by the family $\left\{H_{i} / M\right\}$.

Our main aim in this section is to investigate the topology on $H$ given by the normal subgroups $H_{i}$. From Houghton (1981), the subgroup $H_{i}$ of $H=\pi(Y, y)$ is generated by all path classes $[\beta][\delta][\beta]^{-1}$ for which the image of $\delta$ lies in $Y_{i}$. We shall first consider the case where $Y$ is 1-dimensional and we begin with a combinatorial lemma. 
LEMMA 3. Let $\Gamma$ be an infinite connected locally finite graph. One can choose a chain $\Gamma=\Gamma_{0} \supseteq \Gamma_{1} \supseteq \cdots$ of cofinite subgraphs and a spanning tree $T$ of $\Gamma$ such that $\cap \Gamma_{i}=\varnothing$ and, for all $i$, each intersection of $T$ with a component of $\Gamma_{i}$ is connected.

Proof. We use induction and begin by choosing $y$ in the vertex set $V$ of $\Gamma$ and taking $T_{1}=\{y\}$. For the general step, suppose that $T_{i}$ is a finite tree in $\Gamma$ with vertex set $S_{i}$ and let $\Gamma_{i}$ be the full subgraph of $\Gamma$ based on $V \backslash S_{i}$. In each component of $\Gamma_{i}$ choose a finite tree visiting each vertex adjacent to $T_{i}$ and join this tree by a single edge to $T_{i}$. Applying this procedure to all components of $\Gamma_{i}$, we obtain a tree $T_{i+1}$. The tree $T$ is defined as the union of the $T_{i}$. At each stage of the construction we have incorporated all vertices adjacent to the existing tree and hence $T$ spans $\Gamma$ and $\cap \Gamma_{i}=\varnothing$. For each component $U$ of $\Gamma_{i}$ there is a single edge of $T$ joining $U$ to $T_{i}$ and thus $T \cap U$ is connected.

THEOREM 4. Let $\Gamma$ be an infinite connected locally finite graph. With respect to the topology induced by the map $\pi(\Gamma) \rightarrow \check{\pi}\left(\Gamma^{*}\right)$, the group $\pi(\Gamma)$ is topologically isomorphic to $F^{(\omega)}$. Furthermore, $\check{\pi}\left(\Gamma^{*}\right)$ is topologically isomorphic to $C(X, x)$, where $X$ is the one-point union $E \vee N^{*}$ of the end space $E$ of $\Gamma$ and the one-point compactification of a countably infinite discrete set $N$.

Proof. The fundamental group $F=\pi(\Gamma, y)$ is freely generated by a set bijective with the set of edges of $\Gamma$ not in $T$. For such an edge from $u$ to $v$, the corresponding path class is obtained by going from $y$ to $u$ via $T$, traversing the edge, and returning from $v$ via $T$. For given $i$, the lemma implies that almost all such classes will be of the form $[\beta][\delta][\beta]^{-1}$, with the image of $\delta$ lying in $Y_{i}$. If the free generators of $F$ are denoted arbitrarily by $x_{1}, x_{2}, \ldots$ then a homomorphism of $F$ will be continuous with respect to our topology if and only if almost all $x_{i}$ are mapped trivially. Thus $F$ is topologically isomorphic to $F^{(\omega)}$. Now $\check{\pi}\left(\Gamma^{*}\right)$ is the completion of $F^{(\omega)} * F(E, e)$, where the topology on the free product is precisely that given by its interpretation as $F\left(E \vee N^{*}\right)$. Thus $\pi\left(\Gamma^{*}\right)$ is $C\left(E \vee N^{*}\right)$.

COROllary 5. Let $Y$ be an infinite connected locally finite $C W$-complex with 1-skeleton $\Gamma$. The topology induced on $H=\pi(Y)$ by the homomorphism to $\check{\pi}\left(Y^{*}\right)$ is identical with the topology defined by the epimorphism from $F^{(\omega)}$ to $H$, where $F^{(\omega)}$ is realised as $\pi(\Gamma)$.

Proof. Choosing the $\Gamma_{i}$ as in Lemma 3, we put $Y_{i}^{(1)}=\Gamma_{i}$ and define $Y_{i}$ inductively by attaching to $Y_{i}^{(n)}$ all $(n+1)$-cells with boundary in $Y_{i}^{(n)}$. The sets are cofinite subcomplexes of $Y$ with $\cap Y_{i}=\varnothing$ and the result follows from the previous description of the topology on $H$. 


\section{References}

G. Higman (1952), 'Unrestricted free products, and varieties of topological groups', J. London Math. Soc. 27, 73-81.

C. H. Houghton (1981), 'Homotopy classes of maps to an aspherical complex', J. London Math. Soc. (2) $23,332-348$.

Department of Pure Mathematics

University College

P.O. Box 78

Cardiff, CF1 $1 \mathrm{XL}$

Wales, U.K. 